\title{
Analysis of Instant Messaging Applications
}

\author{
Prinkal \\ Department of Information Technology, Maharaja Agrasen Institute of Technology Delhi, India
}

To Cite this Article

Prinkal, "Analysis of Instant Messaging Applications", International Journal for Modern Trends in Science and Technology, 6(12): 414-417, 2020.

\section{Article Info}

Received on 16-November-2020, Revised on 09-December-2020, Accepted on 12-December-2020, Published on 18-December-2020.

\section{ABSTRACT}

This paper reports on an ongoing project related to Chat Applications. The rapid development of many modern communication technologies have given users many more options for communicating in their workplace and personal life of these new communication technologies, instant messaging is one of the most proliferating communication technologies adopted. Instant messaging protocols have also enabled the users to interact in a more faster way as compared to some other protocols like e-mail. We will study that how we can efficiently integrate real-time chat into the client app.

KEYWORDS: Instant Messaging, communication, chat

\section{INTRODUCTION}

In the era of telecommunication networks, presence information is defined as a status indicator which shows the willingness of other person or communicator to communicate. The client application on the user's side contacts a presence service and provides presence state via network. At the server end it is stored in his personal availability record and is distributed to other users to convey his availability for communication. Modern instant messaging clients use various user availability states such as "free for chat", "busy", "away", "do not disturb" etc. to portray the users current mood, location or free text status. Presence shows the availability state of a user (and maybe much more information about his current state, location etc) and any other user can make use of this data to decide whether to start a conversation with that user or not.

Instant Messaging is a form of real-time communication which is text based between two or more or a group of people with personal computers or any other devices using shared clients, also a user needs to know the presence information of the other person to initiate a messaging session with them. Instant Messaging is considered as one among the various 'online chat' protocols. It differs from e-mails in the sense that Instant Messaging is real-time communication. It allows the user, advantages of both email systems, where communication is free and telephonic systems where communication is real-time. Modern companies try to combine different solutions with Instant Messaging and produce combined and highly potential applications. We will be discussing many of the current Instant Messaging architectures in the next section. It is also possible to save instant messages for a later reference and many current instant messaging/chat systems support this feature. Each modern Instant Messaging generally provides its own client which can be a separately installed software or a browser based client. Also, lately there are many third-party client software applications that will connect with most of major Instant Messaging services. 


\section{Methodology}

Comparison of various Android based Chatting Applications are to be done in this paper on the basis of accuracy metric, Built-in security, and privacy features as large amounts of data are being transmitted over the internet when people make use of these kind of Apps.

The project originated from a popular application called "Telegram", This Application is a cross-platform cloud based Instant Messaging platform which was launched for ios in August 2013 but now It is available for Android, iOS, Windows

Phone, Windows, macOS and GNU/Linux in addition to a web app. Telegram emphasize on sending messages and exchange videos, photos, stickers, audio, and files of any type accurately up to 2 GB each. As all these functionalities and services for the program have been explained, the main structure and construction of the project has been basically illustrated with its goals.

\section{Related Works}

Messaging Apps like Telegram and WhatsApp are well known and very popular multi platform Messaging services. The Headline feature of both of these is Privacy, and to ensure this it employs end-to-end encryption which stops those outside a two-way conversation be it a company, hackers, or someone unauthorized from seeing what data has been sent.

\section{Telegram}

Telegram is a cross-platform multi platform Instant Messaging service founded by "Pavel Durov" a Russian entrepreneur for ios in August 2013.

Telegram is not a just another Messaging app. Although, It's core functionalities like messaging other Telegram users, create group chats, calls, and send files and stickers. is same as most other messaging apps. Telegram uses encryption in calls and it's "Secret chat" which doesn't means it is more secure and private than WhatsApp. Both services uses two factor authentication.

According to the FAQ page of Telegram, It is said that the company is funded by the Founder and
CEO "Pavel Durov" and not through data sharing, collection and advertisements. In other words Telegram provides the Internet privacy as protecting your personal data from Third party access such as marketers, advertisers etc.

Telegram offers excellent features like Self-destruct timers, Global message deletion, Large file size limit. Telegram is designed as a light app, reliable and fast. Telegram also provides bots which is an automatic answering account that can respond to specific text command by answering with preformatted text.

\section{WhatsApp}

WhatsApp, is an American freeware, cross-platform messaging an d Voice over IP service owned by the giant Facebook. WhatsApp was founded by Brian Acton and Jan Koum in September 2007 for Android.

WhatsApp is the most engaged top social messaging application. It is a well known fact that most of the

smartphone users uses WhatsApp messenger. If there can be a competent for WhatsApp, in all probabilities then it will be the Telegram. But there are some freaks, geeks cult prefer Telegram than WhatsApp. Initially WhatsApp was criticized for lack of encryption and sending messages as plain text. Encryption in WhatsApp was first added in May 2012. In 2016, WhatsApp was widely praised for the addition of end-to-end encryption and earned a 6 out of 7 points on the Electronic Frontier Foundation's "Secure Messaging Scorecard".

Just like Apple's iMessage and Signal, WhatsApp has a number of advanced security features like end-to-end encryption. WhatsApp couldn't read your messages as all the messages are secured so that only the sender and receiver can view them. WhatsApp is gaining more populariry than it's competetors like facebook, messenger which is it's own sibling and Telegram as telegram doesn't supports Video Calling. 


\section{RESULTS}

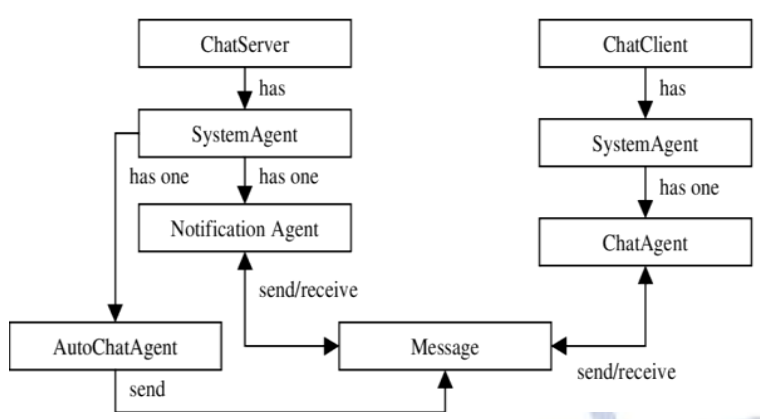

Figure 3 Development process 1
The Flow Chart describes the development process that include all the phases in the software development life cycle. This chart illustrates very well how the project is carried out and how the development was managed.

After the program is completed, the program still needs future maintenance to form it available and stable to execute. The program are going to be tested after a particular period of time and debug each of the function and possible bugs, whenever a possible bug is detected; the program may need to be refined to fix the bugs for better design. Meanwhile, there will be updates, enhancements and more add on to the database to increase the database capacity.

This project's main focus is on privacy: messages shared between users should be encrypted to maintain privacy; Robustness: In case users device crashes, a backup of their chat history must be stored on remote database servers to enable recoverability and performance application must be light weighted.

\section{DISCUSSION}

As this project provides a platform to initiate a real-time chat. On the server side it ensures the reliable infra-management services for chat within the app.

This application or messaging system aims to provide a platform for two individual users separated by a certain geographical distance to communicate with each other, through the Internet with the help of various Firebase tools. In firebase you don't need to write the server side code. Data in the Firebase real-time database is always stored as key-value pairs. FirebaseUI uses a very class named FirebaseListAdapter, which dramatically reduces the effort required to display a ListView using data which present in the
Firebase real-time database. It will be used to fetch and display all the Chat Message objects that are present in the database of firebase.

The minimum requirements for this Android App are:

- Android 4.0 (API level 14) or higher

- Java 7 or higher

- Gradle version 3.4.0 or higher

It is very simple to implement the chat, firstly a user logs in and sees a list of channels then he can select or creates a channel, and sends a message to the channel while receiving messages from other users and communicators within the channel.

In this project, Two types of channels are present: open and group. Open channel is a public channel and anyone can participate in and chat with others. And the group channel is a private channel that users can join as new members through invitation only, and has numerous distinctive properties and features compared to an open channel. There are a variety of group channel subtypes such as a public group channel working like an open channel as shown in fig. 1.
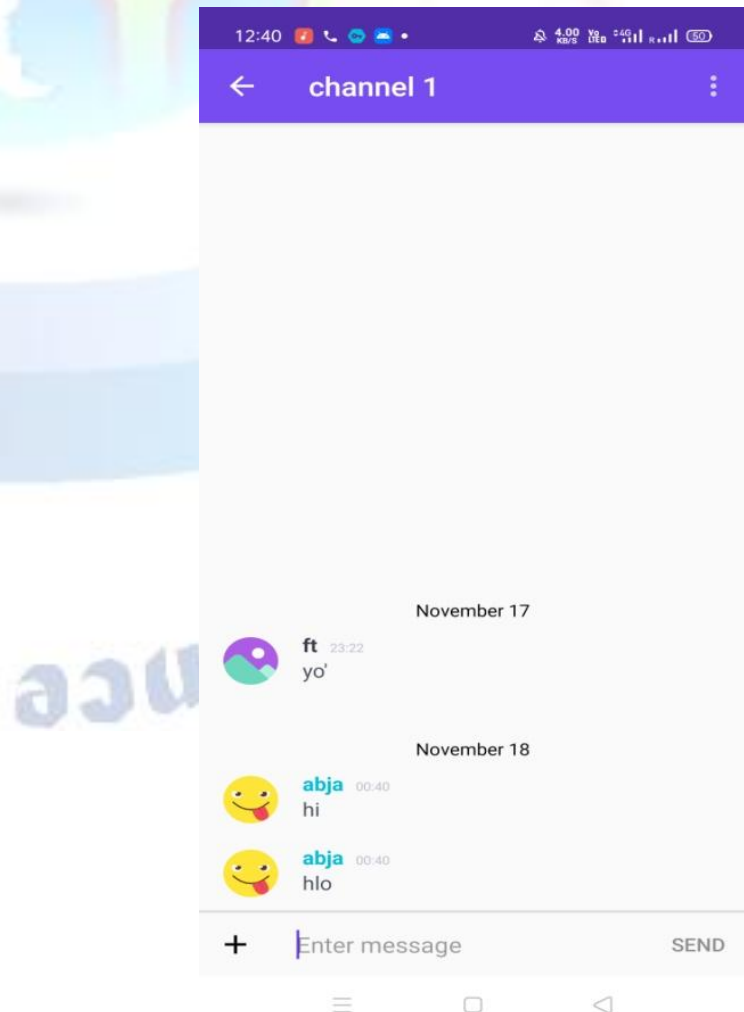

Figure 1 Chat in open channel 
We have also intended the Delivery receipt feature(Double tick) which indicates whether a message has successfully been delivered to all the recipients. Delivery receipt works in a similar way as WhatsApp to read receipt. The server stores the timestamp of the message last delivered. The timestamp is then recorded per user and per channel. When a message is delivered to an online group channel, it is automatically marked as delivered and the other online members are notified of delivery receipt.

The project is very useful and because of the flexible nature of the Firebase tools, the application is a step forward in the right direction in the context of Instant Messaging.

On completion of this system, we are left with a fully functional Instant messaging application capable of sending messages in real-time and images were also being transferred. The Firebase services are important in the sense that those tools made the development of this applications a lot more efficient and lot faster compared to building a traditional server-side database using a scripting language. The application is user-friendly and intuitive, so using it is not a difficult task.

No program has a perfect design without any flaws, it is same here in this project. Even though the program includes the primary functions implemented and working properly, there are still many things that can be done to improve its performance.

\section{REFERENCES}

[1] Bill Philips, Chris Stewart, Kristin Marsicano. Android Programming: The Big Nerd Ranch Guide. 3rd Edition. Arizona: Big Nerd Ranch; 2017.

[2] Kumar, S., et al. Embedded end-to-end wireless security with ECDH key exchange. in Circuits and Systems, 2003 IEEE 46th Midwest Symposium on. 2003: IEEE.

[3] Dashtinejad, P., Security System for Mobile Messaging Applications. 2015

[4] Cortjens, D., Spruyt, A. \& Wieringa., W. F. C., n.d. "WhatsApp Database Encryption Project, s.1.: s.n.

[5] Thakur, N., 2013. Forensic Analysis of WhatsApp on Android Smartphones.. Master's thesis, University of New Orleans, , Volume 1706.

[6] Barghuthi, N. A. \& Said., H., 2013. Social Networks IM Forensics: Encryption Analysis.. Journal of Communications, $8(11)$ 